\title{
Diagnostic criteria and problems in infective endocarditis
}

\section{B D Prendergast}

Heart 2004;90:61 1-613. doi: 10.1136/hrt.2003.029850

Few diseases present greater difficulties in the way of diagnosis than malignant endocarditis, difficulties which in many cases are practically insurmountable. It is no disparagement to the many skilled physicians who have put their cases upon record to say that, in fully one-half the diagnosis was made post mortem. - William Osler 1885

O sler's portentous words are as relevant now as when originally published. Despite improved preventive strategies, rational antibiotic prescribing, advances in imaging, and increasing use of early life saving cardiac surgery, the incidence of infective endocarditis remains high at 1.7-6.2 per 100000 person years in the USA and Europe, with a one year mortality approaching $40 \%{ }^{1}$ The classical patient with infective endocarditis described in textbooks no longer represents the majority of cases in practice. The emergence of staphylococcal infection, often associated with indwelling devices, co-existent medical conditions and resistant to conventional antibiotic regimes, a variety of other atypical organisms, and the persistent syndrome of culture negative endocarditis make diagnosis and treatment as great a challenge as ever.

\section{DIAGNOSTIC CRITERIA AND THEIR LIMITATIONS}

The original von Reyn diagnostic criteria for infective endocarditis, ${ }^{2}$ based upon clinical and microbiological features, have now been surpassed by the Duke criteria ${ }^{3}$ which emphasise the role of echocardiography, the key imaging tool for both diagnosis and assessment of prognosis. Many studies have now demonstrated the superiority of the Duke criteria and a recent scientific statement of the American Heart Association concluded that they should be adopted as the primary diagnostic schema in the clinical evaluation of patients in whom infective endocarditis is suspected. ${ }^{4}$ Nevertheless, clear deficiencies remain. Thus, in one series ${ }^{5}$ of 93 patients with pathologically confirmed infective endocarditis (affecting a native valve in 63 and a prosthetic valve in the remainder), 22 were misclassified as "possible" cases using the Duke criteria, yielding a sensitivity of only $76 \%$. Echocardiographic major criteria were present in 19 patients but blood cultures were negative in 21 . The cause of negative blood cultures was prior antibiotic treatment in 11 patients and Q fever endocarditis detected by positive serology in three. The Duke criteria have also been shown to be of lower value in other important patient groups, including those with infection affecting a prosthetic valve or pacemaker lead and the right heart in drug abusing patients.

\section{CULTURE NEGATIVE INFECTIVE ENDOCARDITIS AND ATYPICAL ORGANISMS}

Negative blood cultures occur in $2.5-31 \%$ of all cases of infective endocarditis, often delaying diagnosis and the onset of treatment with profound impact on clinical outcome. Moreover, the sensitivity of the Duke criteria, which rely heavily on microbiological indices, is diminished in this setting. Negative cultures arise most commonly as a consequence of prior antibiotic administration, but an increasingly common scenario is infection by fastidious organisms with limited proliferation under conventional culture conditions, or requiring specialised tools for identification. Such pathogens include Coxiella, Legionella, the HACEK group (Haemophilus species, Actinobacillus actinomycetemcomitans, Cardiobacterium hominis, Eikenella corrodens, and Kingella kingae), Chlamydia, Bartonella, Tropheryma whippelii, and fungi, including Candida, Histoplasma, and Aspergillus species, and Torulopsis glabrata. These organisms may be particularly common in cases of infective endocarditis affecting patients with prosthetic valves, indwelling venous lines, pacemakers, renal failure, and immunocompromised states.

\section{MODIFIED CRITERIA AND NEW DIAGNOSTIC TECHNIQUES}

In 1997, Lamas and Eykyn proposed a number of clinical modifications to the Duke criteria to include newly diagnosed splenomegaly or clubbing, elevated inflammatory markers, haematuria, and the presence of central and peripheral venous lines ("the St Thomas modifications"). ${ }^{6}$ Simultaneously, recognition of the role of Q fever, a worldwide zoonosis caused by Coxiella burnetti and a particularly frequent cause of infective endocarditis in France, increasing prevalence of staphylococcal infection, and widespread use of transoesophageal echocardiography resulted in further modifications; these involved incorporating the acceptance of positive Q fever serology or bacteraemia caused by Staphylococcus aureus (regardless of its source) as major criteria and the elimination of minor echocardiographic criteria (table 1$).{ }^{78}$ A recent study compared these modifications in pathologically proven yet culture negative cases. ${ }^{9}$ Only $21 \%$ were classified as definite by the original Duke criteria, while $32 \%$ were definite by the modified Duke criteria, and the St Thomas modifications classified $62 \%$ correctly. Interestingly, the four additional cases identified correctly by the modified Duke criteria were upgraded on account of positive Q fever serology. Furthermore, careful scrutiny led to identification of the causative organism in almost $50 \%$ of culture negative cases, achieved by serology (C burnetti, Bartonella, Chlamydia psittaci) in $24 \%$, culture of the excised valve in $14 \%$, microscopy of the excised valve in $5 \%$, and non-valve culture in $6 \%$.

\section{Histological/immunological techniques}

Histological findings are included in the Duke and von Reyn diagnostic criteria and pathological examination of resected valvar tissue or embolic fragments remains the gold standard for the diagnosis of infective endocarditis. Pathological examination may also guide antimicrobial treatment if the causative agent can be identified by means of special stains or immunohistological techniques, and allow distinction from conditions whose echocardiographic features mimic infective endocarditis (for example, myxomas, fibroelastomas, and rheumatoid nodules). It should be noted that specimens are potentially infectious to the examiner, particularly when Q fever is suspected. The pathological hallmark of endocarditis 
Table 1 Duke criteria for the diagnosis of infective endocarditis and proposed modifications

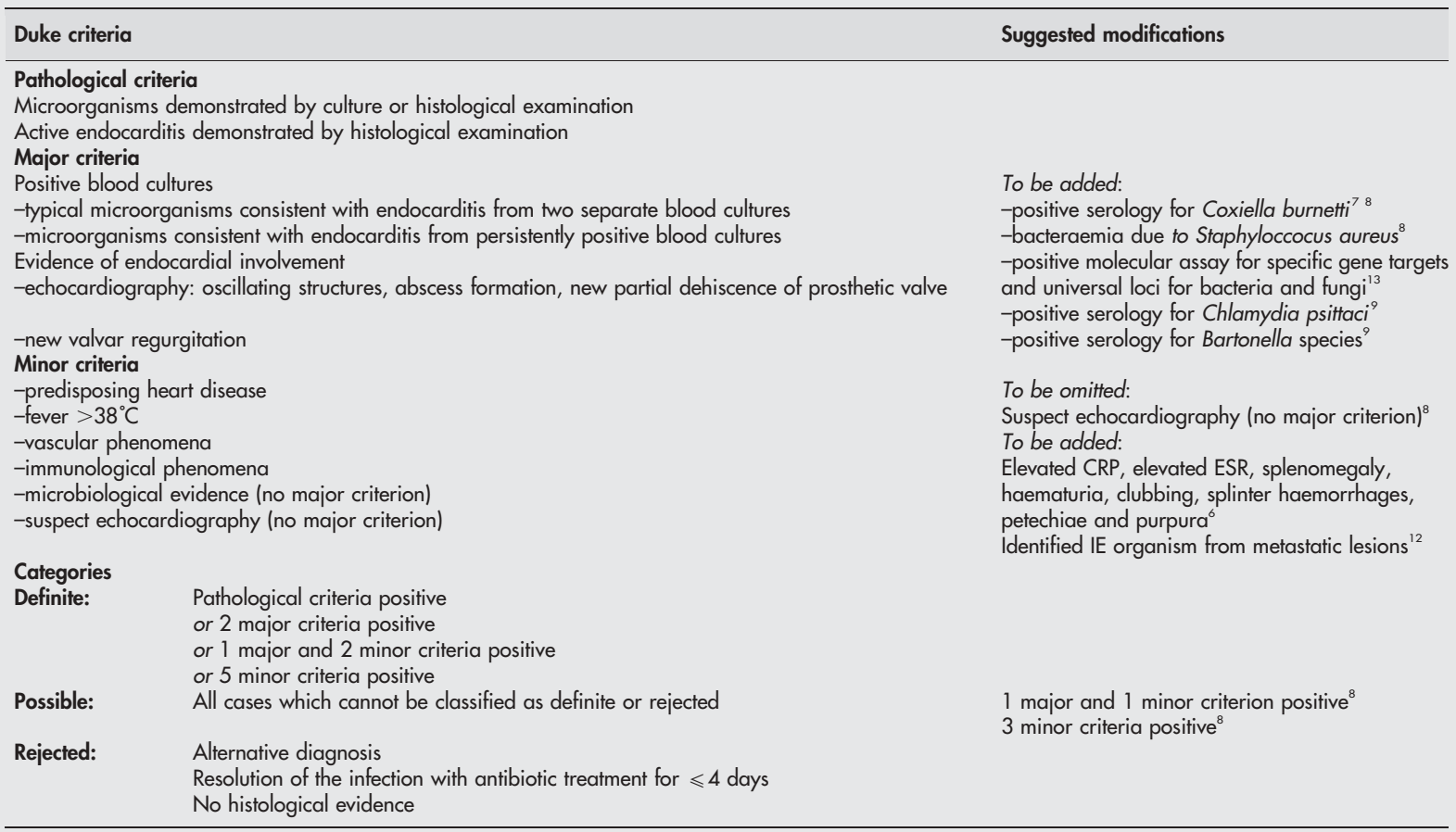

CRP, $C$ reactive protein; $E S R$, erythrocyte sedimentation rate; $I E$, infective endocarditis.

Adapted with permission from Naber CK, Erbel R. Heart 2003;89:241-3.

is the demonstration of inflammatory changes in valvar tissue and/or vegetations, characteristically at the site of attachment or base of a vegetation. This finding is not specific, however, and inflammation is also a feature of degenerative and other valve pathology. Electron microscopy has high sensitivity and may help to characterise new microorganisms. However, it is time consuming and expensive and therefore reserved for cases in which other techniques fail to detect an organism. Histological criteria for the diagnosis of endocarditis have been proposed (table 2) and a variety of specialised stains and immunohistological techniques (immunoperoxidase staining, enzyme linked immunosorbent (ELISA) and immunofluorescent (ELIFA) assays, and direct immunofluorescence using fluorescein conjugated monoclonal antibodies) are now available to allow the identification of elusive bacteria and fungi (table 3). ${ }^{10}{ }^{11} \mathrm{C}$ burnetti and Bartonella species are the most common aetiological agents in culture negative endocarditis and may be easily detected by serological testing using indirect immunofluorescence or ELISA. Immunological analysis of urine may also allow detection of microorganism degradation products and ELISA detection of Legionella species has been described using this technique. However, the incorporation of these techniques into accepted diagnostic criteria awaits prospective validation.

\section{Molecular techniques}

Several molecular approaches have been assessed for the detection and identification of pathogens in a wide variety of infectious diseases. The polymerase chain reaction (PCR) utilising nucleic acid target or signal amplification, alone or in combination with sequence analysis, is most widely used and allows rapid and reliable detection of fastidious and nonculturable agents in blood and surgical material of patients with infective endocarditis. ${ }^{12}$ It may also be of value when phenotypic characterisation is essential following isolation of two or more organisms in separate cultures (most commonly caused by contamination with skin commensals during sampling or polymicrobial infection in intravenous drug abusers). Indeed, the incorporation of such techniques as a major Duke diagnostic criterion has been proposed with widespread support. ${ }^{13}$ Specific primers are now available

Table 2 Proposed histological criteria for diagnosis of infective endocarditis

\begin{tabular}{l} 
Major criteria \\
Vegetation \\
Active endocarditis, including inflammatory infiltrates with polymorphonuclear cells \\
Microorganisms demonstrated in tissues by histology or immunohistology \\
Minor criteria \\
Mononuclear cell infiltrate (macrophages and lymphocytes) of the valve \\
Necrosis \\
Neovascularisation \\
Fibrosis \\
Calcification \\
Definite IE: 2 major or 1 major and 3 minor criteria \\
Possible IE: 1 major and 2 minor criteria \\
Rejected IE: No major criterion \\
\hline IE, infective endocarditis. \\
Reproduced from Lepidi et al ${ }^{10}$ with permission.
\end{tabular}


Table 3 Main histological stains used for the diagnosis of infective endocarditis

\begin{tabular}{ll}
\hline Tissue stain & Detected microorganism \\
\hline Acridine orange & Any bacterium \\
Giemsa & Any bacterium \\
Tissue Gram & \\
Brown-Hoops & Gram positive bacteria \\
Brown-Brenn & Gram negative bacteria \\
Periodic acid-Schiff & Tropheryma whippelii, fungi \\
Warthin-Starry & Bartonella species \\
Ziehl-Nielsen & Acid-fast bacilli \\
Gimenez & Coxiella burnetti, Legionella species \\
Kinyoun, Mchiavello & Chlamydia species \\
Gomori-Grocott & Fungi \\
\hline
\end{tabular}

Reproduced from Houpikian et al ${ }^{1}$ with permission.

for many bacterial agents including $T$ whippelii, $C$ burnetti, and species of Bartonella, Chlamydia, Brucella, Legionella, Mycobacteria, and Mycoplasma. Future improvements include the possibility of quantitation by real time PCR eliminating the need for gel electrophoresis with faster, more accurate results, and the possibility of investigating common antimicrobial resistance genes enabling a targeted approach to antibiotic treatment. ${ }^{14}$

In a recent validation study of these molecular methods, Grijalva and colleagues ${ }^{15}$ reported the investigation of valvar specimens of 15 patients with definite, though culture negative, infective endocarditis undergoing surgery. The causative organism was identified in 14 (93\%) of the culture negative cases (streptococci 3, staphylococci 2, enterobacter 1, T whippelii 1, Borrelia burgdorferi 1, Candida albicans 1, Aspergillus species 2, unspecified 3) while 13 matched controls yielded negative results. Analysis was complete within 8 hours and within 48 hours if sequencing was required.

\section{PROPOSED APPROACH}

Optimal antiseptic skin preparation is important and at least $10 \mathrm{ml}$ of blood (less in children) should be obtained for each culture. Bacteraemia is usually continuous and there is no rationale for timing blood cultures to coincide with peaks of pyrexia. If there is a history of prior antibiotic treatment, maximum diagnostic yield may be achieved by diluting the culture broth and considering the use of sodium polyanetholsulfonate or a dedicated adsorbent resin, both of which inactivate antimicrobial effects. Multiple separate blood cultures are required and most guidelines recommend at least two, and most usually three sets. Although endocarditis caused by anaerobes is uncommon, blood cultures should be incubated in both aerobic and anaerobic atmospheres to detect organisms such as Bacteroides or Clostridium species.

If all blood cultures remain negative at five days but infective endocarditis remains likely on clinical grounds, subculture onto chocolate agar plates may allow identification of an atypical organism, although prolonged culture for up to 2-3 weeks is associated with rising likelihood of contamination. At this stage, therefore, serum should be analysed for antibodies to organisms that cannot be cultured by routine methods, and, if available, antibodies to Gram positive bacterial cell walls. Excised valves, vegetations or other relevant material should undergo microscopy, culture, histopathology, and relevant molecular techniques (usually PCR) which can also be applied to newly acquired blood samples or the original growth negative blood culture substrate.

\section{CONCLUSIONS}

To date, definitive studies of infective endocarditis have been difficult to perform because of its heterogenous nature. Launched in 1999, the International Collaboration on Endocarditis was conceived to develop a large global database of patients whose clinical, echocardiographic, and microbiological findings have been characterised using standard methodology. The associated network of investigators and organisational infrastructure will provide the platform for large randomised trials to test therapeutic strategies. ${ }^{16}$ This resource offers the opportunity for major advances in our understanding and treatment of infective endocarditis over the next two decades.

Modification of the original Duke criteria is now proposed to enhance diagnostic sensitivity, especially in culture negative cases. Increased emphasis on symptoms and signs ${ }^{6}$ coupled with improved likelihood of identification of a causative pathogen using serology, ${ }^{78}$ additional culture, ${ }^{11}$ or newer histological ${ }^{1011}$ and molecular techniques $^{12}{ }^{13}$ will improve the sensitivity of the diagnosis and increase therapeutic specificity. These measures, combined with close collaboration and communication between the cardiologist, cardiac surgeon, and microbiologist, are essential to ensure optimal diagnosis and management and a favourable impact on patient outcome.

Correspondence to: Dr Bernard D Prendergast, North-West Regional Cardiothoracic Centre, Wythenshawe Hospital, Southmoor Road, Manchester M23 9LT, UK; bernard.prendergast@smuht.nwest.nhs.uk

\section{REFERENCES}

1 Cabell $\mathrm{CH}$, Jollis JG, Peterson GE, et al. Changing patient characteristics and the effect on mortality in endocarditis. Arch Intern Med 2002;162:90-4.

2 von Reyn CF, Levy BS, Arbeit RD, et al. Infective endocarditis: an analysis based on strict case definitions. Ann Intern Med 1981;94:505-17.

3 Durack DT, Lukes AS, Bright DK, et al. New criteria for diagnosis of infective endocarditis: utilization of specific echocardiographic findings. Am J Med 1994;96:200-9.

4 Bayer AS, Bolger AF, Taubert KA, et al. Diagnosis and management of infective endocarditis and its complications. Circulation 1998;98:2936-48.

5 Habib G, Derumeaux G, Avierinos J-F, et al. Value and limitations of the Duke criteria for the diagnosis of infective endocarditis. J Am Coll Cardiol 1999;33:2023-9.

6 Lamas C, Eykyn SJ. Suggested modifications to the Duke criteria for the clinical diagnosis of native valve and prosthetic valve endocarditis: analysis of 118 pathologically proven cases. Clin Infect Dis 1997;25:713-9.

7 Fournier PE, Casalta JP, Habib G, et al. Modification of the diagnostic criteria proposed by the Duke endocarditis service to permit improved diagnosis of $Q$ fever endocarditis. Am J Med 1996; 100:629-33.

8 Li JS, Sexton DJ, Mick N, et al. Proposed modifications to the Duke criteria for the diagnosis of infective endocarditis. Clin Infect Dis 2000;30:633-8.

9 Lamas CC, Eykyn SJ. Blood culture negative endocarditis endocarditis: analysis of 63 cases presenting over 25 years. Heart 2003;89:258-62.

10 Lepidi H, Durack DT, Raoult D. Diagnostic methods. Current best practices and guidelines for histologic evaluation in infective endocarditis. Infect Dis Clin N Am 2002;16:339-61.

11 Houpikian P, Raoult D. Diagnostic methods. Current best practices and guidelines for identification of difficult-to-culture pathogens in infective endocarditis. Infect Dis Clin N Am 2002;16:377-92.

12 Lisby G, Gutschik E, Durack DT. Molecular methods for diagnosis of infective endocarditis. Infect Dis Clin North Am 2002;16:393-412.

13 Millar BC, Moore JE, Mallon P, et al. Molecular diagnosis of infective endocarditis-a new Duke's criterion. Scand J Infect Dis 2001;33:673-80.

14 Moore JE, Millar BC, Yongmin X, et al. A rapid molecular assay for the detection of antibiotic resistance determinants in cause of infective endocarditis. J Appl Microbiol $2001 ; 90: 719-26$.

15 Grijalva M, Horvath R, Dendis $M$, et al. Molecular diagnosis of culture negative infective endocarditis: clinical validation in a group of surgically treated patients. Heart 2003;89:263-8.

16 Cabell CH, Abrutyn E. Progress toward a global understanding of infective endocarditis. Early lessons from the International Collaboration on Endocarditis investigation. Infect Dis Clin North Am 2002;16: 255-72. 